\title{
BMJ Open Furosemide versus mannitol in Japanese patients with thoracic malignancy who received cisplatin-based chemotherapy using short hydration: study protocol for a randomised controlled trial
}

To cite: Murakami E,

Akamatsu H, Shimokawa T, et al. Furosemide versus mannitol in Japanese patients with thoracic malignancy who received cisplatin-based chemotherapy using short hydration: study protocol for a randomised controlled trial. BMJ Open 2019;9:e029057. doi:10.1136/ bmjopen-2019-029057

- Prepublication history for this paper is available online To view these files, please visit the journal online (http://dx.doi org/10.1136/bmjopen-2019029057).

Received 21 January 2019 Revised 26 November 2019 Accepted 27 November 2019

Check for updates

(C) Author(s) (or their employer(s)) 2019. Re-use permitted under CC BY-NC. No commercial re-use. See rights and permissions. Published by BMJ.

${ }^{1}$ Internal Medicine III, Wakayama Medical University, Wakayama, Japan

${ }^{2}$ Clinical Study Support Center, Wakayama Medical University Hospital, Wakayama, Japan

${ }^{3}$ Department of Nursing, Wakayama Medical University, Wakayama, Japan

\section{Correspondence to}

Dr Hiroaki Akamatsu;

h-akamat@wakayama-med. ac.jp

\section{ABSTRACT}

Introduction Cisplatin (CDDP) is a key drug for various thoracic malignancies. To avoid renal toxicity of CDDP, mannitol is routinely used, but it sometimes causes phlebitis which damages patients' quality of life. Furosemide is another widely used option for diuresis administered more quickly. To date, previous comparisons of these diuretics have lacked statistical significance owing to study design. We therefore undertake a randomised phase II comparative study of furosemide and mannitol in CDDP-based chemotherapy using short hydration.

Methods and analysis This is a two-arm, prospective, randomised, single-centre, open-label phase II study. The primary endpoint is set as the proportion of patients who experienced any grade of 'creatinine increase' using the Common Terminology Criteria for Adverse Events V.4.0, during the first cycle. Secondary endpoints are: the proportion of patients who experienced $\geq$ grade 2 of creatinine increase during the first cycle, any grade and $\geq$ grade 2 of creatinine increase after the completion of fourth cycle, and the proportion of patients with phlebitis. Enrolled in this trial will be 105 patients.

Ethics and dissemination This study was approved by the Wakayama Medical University Institutional Review Board on 30 March 2018 study (approval number: 2258). Patients have been enrolled since May 2018. As the study will complete accrual in March 2021, results will be published by 2021. This study will provide important information about the utility of furosemide compared with mannitol to protect against nephrotoxicity.

Trial registration number UMIN000031910.

\section{INTRODUCTION}

Cisplatin (CDDP) is a key drug for various types of thoracic malignancies, but in about $30 \%$ of the patients who receive it there is renal toxicity. ${ }^{1} \mathrm{CDDP}$ causes nephrotoxicity at the S3 segment of the proximal tubules situated in the outer stripe of outer medulla. ${ }^{2}$ To avoid renal toxicity of CDDP, adequate hydration and diuresis are important. ${ }^{34}$ Although
Strengths and limitations of this study

- This is a randomised phase II study that compares mannitol with furosemide for patients with thoracic malignancy who received cisplatin-based chemotherapy using short hydration.

- This is the first statistically well-designed study to assess the utility of furosemide to prevent renal toxicity in chemotherapy compared with mannitol.

- In addition, phlebitis caused by mannitol is a clinically important issue for patients, therefore we will evaluate the incidence and severity of phlebitis as a secondary endpoint.

- Although this is an open-label, single-institutional phase II study, results obtained from 105 participants will be the most powerful evidence in this area.

the mechanism of diuresis to reduce renal toxicity is not exactly known, it is assumed that diuresis prevents CDDP from remaining within renal tubules for a long time. For increased urinary excretion, mannitol, an osmotic diuretic, raises osmotic pressure at the lumen of the tubules. Furosemide, meanwhile, is a loop diuretic and blocks Na-K-Cl cotransporter at the ascending limb of the loop of Henle. Currently, mannitol is the only drug recommended in National Comprehensive Cancer Network Chemotherapy Order Templates (NCCN Templates). On the other hand, furosemide is a widely used option for diuresis. ${ }^{5}$ Several studies have compared the renal toxicity of mannitol and furosemide. A small randomised study (22 patients) by Ostrow et $a l^{6}$ showed that renal toxicity was milder in the furosemide arm $(19 \%$ vs $28 \%){ }^{6}$ In another study (49 patients), decrease in creatinine clearance was smaller in the furosemide arm than in the mannitol arm. ${ }^{7}$ These 


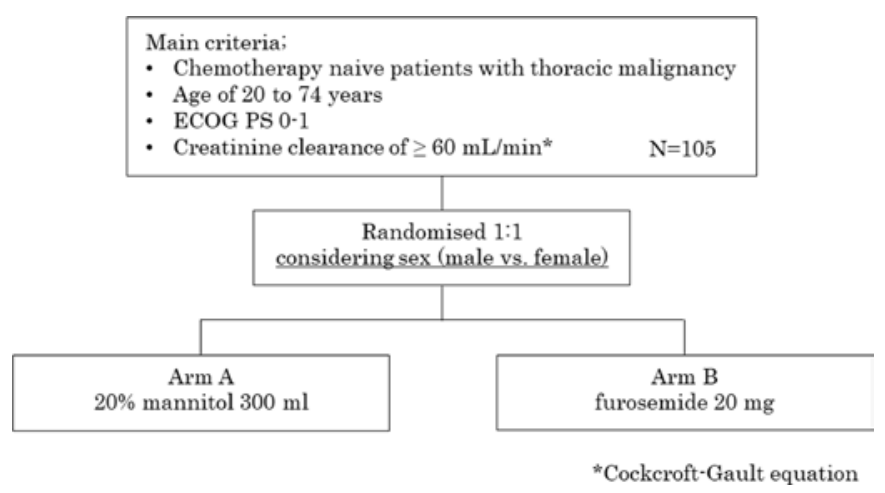

Figure 1 Protocol scheme of this study. ECOG, Eastern Cooperative Oncology Group. PS, performance status.

studies did not have sufficient power, however, to detect statistically significant difference. Regarding toxicity, we often encounter phlebitis, especially in mannitol administration. In terms of efficacy and safety, furosemide may therefore be non-inferior to mannitol. We therefore undertake a randomised phase II comparative study of furosemide and mannitol in CDDP-based chemotherapy using short hydration.

\section{AIM OF THE STUDY}

The main objective of this study is to assess the utility of furosemide compared with mannitol to protect against renal toxicity in CDDP-based chemotherapy ( $\geq 60 \mathrm{mg}$ / $\mathrm{m}^{2}$ ) using short hydration in chemotherapy-naive patients with thoracic malignancy.

\section{STUDY DESIGN}

This study is designed as a two-arm, prospective, randomised, single-centre, open-label phase II study. The protocol scheme is shown in figure 1 . Study duration will be 3 years and 10 months.

\section{Endpoints}

Primary endpoint is set as the proportion of patients who experience any grade of 'creatinine increase' using the Common Terminology Criteria for Adverse Event (CTCAE) V.4.0 (based on the upper limit of the normal (ULN) range for serum creatinine), during the first cycle. Secondary endpoints are: the proportion of patients who experience $\geq$ grade 2 of creatinine increase (based on the ULN for serum creatinine) during the first cycle, any grade and $\geq$ grade 2 of creatinine increase (based on the pretreatment baseline creatinine score in each patient) during the first cycle, any grade of creatinine increase (based on both criteria) after the completion of fourth cycle, and the proportion of patients who had phlebitis.

\section{Eligibility criteria of the participants}

Inclusion criteria

Patients are required to fulfil all the following criteria:

1. Histologically or cytologically confirmed thoracic malignancy.
2. Aged between 20 and 74 years.

3. Eastern Cooperative Oncology Group performance status of $0-1$.

4. Adequate renal function (including both serum creatinine $\leq 1.2 \mathrm{mg} / \mathrm{dL}$ and a creatinine clearance of $\geq 60 \mathrm{~mL} / \mathrm{min}$ using Cockcroft-Gault equation).

5 . Tolerable of oral hydration $\geq 1 \mathrm{~L}$ per day.

6. Normal cardiac function.

7. Written informed consent.

Exclusion criteria

Patients are excluded if they meet any of all the following criteria:

1. Superior vena cava syndrome.

2. Active mental illness.

3. Pregnancy, breast feeding, or possibility of being pregnant.

4. Other conditions rendering patients unsuitable for this study, for example, serious complications (severe heart disease, gastrointestinal bleeding, glaucoma, etc).

\section{Discontinuation criteria}

Interventions are discontinued if patients meet any of all the following criteria:

1. Disease progression or death.

2. Serum creatinine $>2.0 \mathrm{mg} / \mathrm{dL}$ or unacceptable toxicity.

3. Patient refusal.

4. Other reasons of unsuitability owing to which the study protocol should not continue.

\section{Background data}

Patient background data are collected prospectively for all patients including sex, age, history, complications, allergies, past treatments for cancer and concomitant chemotherapy.

\section{Interventions}

Patients receive CDDP-based chemotherapy $(\geq 60 \mathrm{mg} /$ $\mathrm{m}^{2}$ ). After common antiemetic premedication (aprepitant, palonosetron and dexamethasone) and one other cytotoxic agent, an hour-long infusion of CDDP dissolved in a $500 \mathrm{~mL}$ of normal saline solution is administered between the prehydration (potassium chloride and magnesium sulfate dissolved in $500 \mathrm{~mL}$ of normal saline solution) and posthydration $(500 \mathrm{~mL}$ of maintenance solution). In arm $\mathrm{A}$, patients receive $300 \mathrm{~mL}$ of $20 \%$ mannitol by intravenous drip infusion over $30 \mathrm{~min}$ just before CDDP. In arm B, patients receive $20 \mathrm{mg}$ of furosemide intravenously an hour before CDDP (figure 2). These doses and schedules are basically in accordance with NCCN templates and previous studies. In addition, on day 2 and 3, patients are orally or intravenously administered dexamethasone $8 \mathrm{mg}$ per day. CDDP-based chemotherapy is repeated every 3 or 4 weeks for up to four cycles except where there is disease progression, unacceptable toxicity, or patients' refusal. 


\begin{tabular}{|l|l|}
\hline \multicolumn{1}{|c|}{ Arm A (mannitol) } & \multicolumn{1}{c|}{ Arm B (furosemide) } \\
\hline \begin{tabular}{l|l|} 
Antiemetic-1 $(30 \mathrm{~min})$ \\
Aprepitant $150 \mathrm{mg}$ \\
Normal saline solution $250 \mathrm{ml}$
\end{tabular} & $\begin{array}{l}\text { Antiemetic-1 }(30 \mathrm{~min}) \\
\text { Aprepitant } 150 \mathrm{mg}\end{array}$ \\
Normal saline solution $250 \mathrm{ml}$
\end{tabular}

Figure 2 Details of each regimen. CDDP, cisplatin.

\section{Supportive therapy}

Patients are recommended to use the following therapy. Even if this therapy is not used, patients are not considered to have dropped out.

1. Oral hydration of approximately $1 \mathrm{~L}$ by CDDP completed.

2. Additional use of antinausea therapy (olanzapine, metoclopramide, etc) if patients reported nausea (olanzapine is optional therapy because it is not yet approved in Japan).

\section{Follow-up and assessment}

Blood test data (including complete blood cell, electrolytes $(\mathrm{Na}, \mathrm{K}, \mathrm{Ca})$, creatinine and creatinine clearance based on Cockcroft-Gault equation) are evaluated on day 1 of every cycle, on day 8 of the first cycle, and within 30 days after the last administration of CDDP. We measure amounts of drinking and of urine during days 1-5 of the first cycle. Furthermore, we evaluate additional rehydration from day 1 of the first cycle to the day before the start of the second cycle. Phlebitis is evaluated on days 1-5 and day 8 in cycle 1 and on day 1 of all other cycles thereafter in both arms. Phlebitis is assessed by at least two medical staff (a nurse and another staff physician). Adverse events (all grades of anorexia, nausea, vomiting, diarrhoea, fatigue and other adverse events grade 3 or higher) are evaluated on day 1 of every cycle, on day 8 of the first cycle (table 1). All adverse events are defined according to the CTCAE V.4.0.

\section{STATISTICAL ANALYSIS}

The primary population for efficacy analysis is the intention-to-treat population, defined as all randomised patients. The primary endpoint is the proportion of patients who experienced any grade of renal dysfunction. It will be evaluated using risk difference (arm Barm A) with $80 \%$ CIs (one-sided). An upper confidence limit below $10 \%$ suggests that arm B is non-inferior to arm A. The proportion of renal dysfunction for each arm is summarised descriptively with Clopper and Pearson's exact 95\% CIs. The stratified OR is estimated from logistic regression analysis with randomised factors as covariates. Moreover, multivariate regression analysis is used to explore interactions between therapies and background factors. Secondary endpoints are summarised using frequency and percentage with exact 95\% CIs using Clopper and Pearson's method for each arm. Missing data will be not imputed and will be excluded from the analysis of the corresponding endpoint.

\section{Registration and randomisation}

After registration, patients will be randomised at 1:1 ratio, using minimisation method that balances the treatment arms by sex as an allocation factor (male vs female). The randomisation schedule will be prepared and maintained by a registration centre as independent central randomisation.

\section{Sample size calculation}

Using mannitol, two previous prospective studies conducted in Japanese academic centres reported that the proportion of patients who experienced any grade of creatinine increase using the CTCAE V.4.0 (based on the ULN for serum creatinine) was $0 \%-9 \%$ during the first cycle. ${ }^{89}$ Using this information, we assume the proportion of patients who experienced any grade of renal dysfunction in arm A as $10.0 \%$. Sample sizes of 51 patients in each arm achieve $\geq 80 \%$ power to detect a non-inferiority margin difference between the group proportions of $10.0 \%$ using the one-sided binomial test with alpha error of 0.2. The alpha error and power were chosen based on phase II screening studies. Considering that about $5 \%$ of the patients will be censored, a total of 105 patients are required in the present study.

\section{PATIENTS AND PUBLIC INVOLVEMENT}

Patients were not directly involved in construction of this study but this study adopted the occurrence of phlebitis as a secondary endpoint. This idea was taken from patients' claim with mannitol infusion. We therefore believe that patients' priorities and requirements partly affected the study design.

\section{ETHICS AND DISSEMINATION}

The study is designed and implemented according to good clinical practice and conducted in accordance with the Declaration of Helsinki. This study was approved by the Institutional Review Board of Wakayama Medical University on 30 March 2018 (approval number: 2258). Patients have been enrolled since May 2018. As the study will complete accrual in March 2021, results will be published within 2021. This study will provide important information whether furosemide would be clinically useful than mannitol regarding convenience and in 


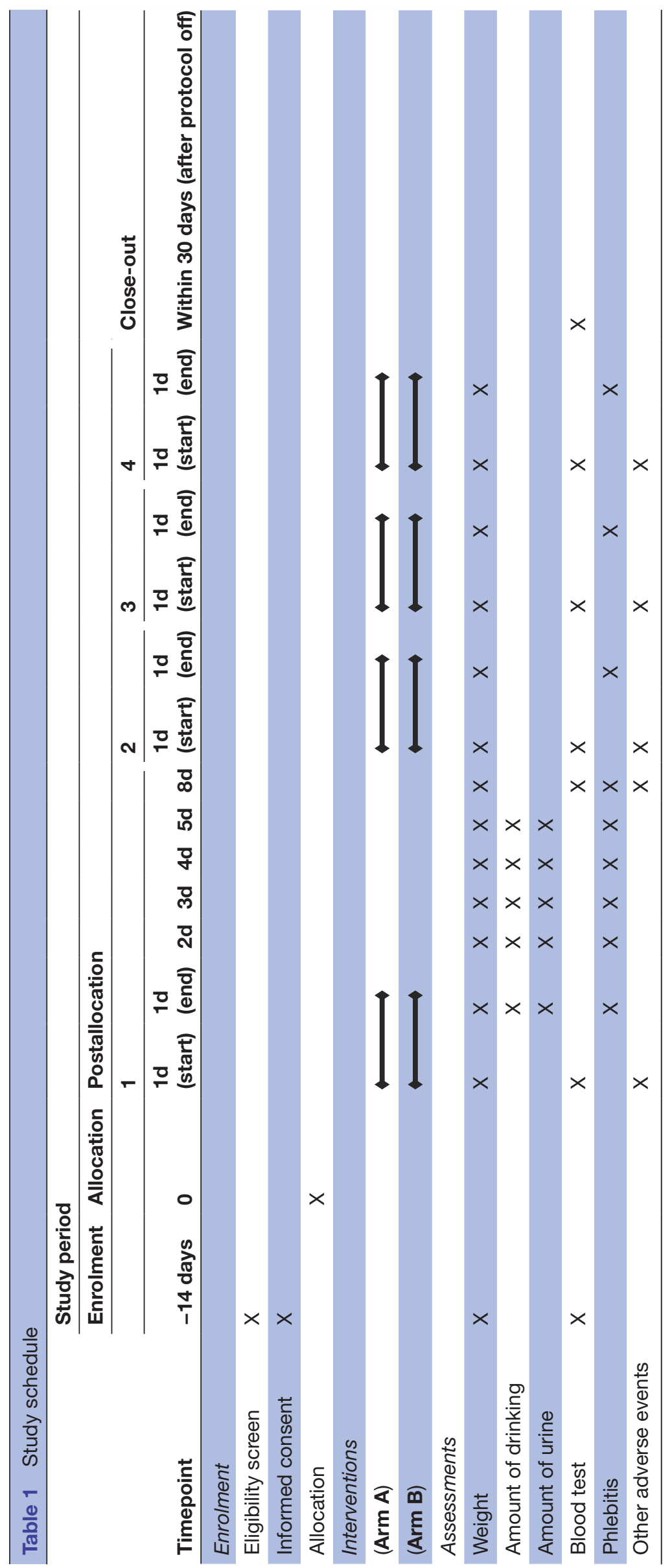

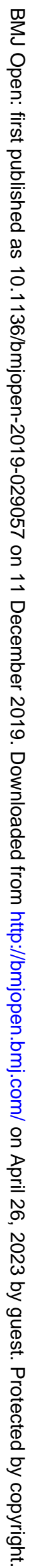


reduction of phlebitis. For participants, results of this study will be announced by their physicians.

\section{INFORMED CONSENT}

All enrolled patients will be informed of the purpose of the study, the method of randomisation, the plan of intervention, the benefits to the participants and the possible risks, after which they will sign the patient consent form. All patients will be given enough time to consider whether they would like to participate in this study. Patients participating in the study will be allowed, at any stage, to freely withdraw their consent form or to withdraw from the study without restrictions.

\section{CONFIDENTIALITY}

Data are collected via datasheets on paper and kept securely. All handling cases are managed by anonymised registration number. The correspondence table of the anonymising code and names and the consent form containing the names are kept strictly in the separate lockable document storage at Internal Medicine III, Wakayama Medical University.

\section{MONITORING}

Monitoring will be performed annually by each dataset to evaluate and improve study progress and quality.

\section{DISCUSSION}

Diuresis is necessary to avoid renal toxicity by CDDP. Although the mechanism of diuretic-induced reduction of renal toxicity has not been fully clarified, the acceleration of urinary excretion leads to shortening of the period of CDDP exposure. Although mannitol is the only drug recommended in NCCN templates, furosemide may be a useful option based on the results of several small studies. The number of participants in these studies was relatively small, however, so their results cannot be evaluated properly. We therefore planned this study to overcome these limitations and it is the first well-designed randomised study in this area. Additionally, we predefined the scheduling blood examinations so the arms would be similar. These considerations will demonstrate the actual utility of furosemide compared with mannitol. We set phlebitis as the secondary endpoint. Although phlebitis is a painful adverse event for patients who receive anti-cancer treatment, its exact incidence has not been described. Furthermore, how much mannitol may provoke phlebitis has not been clarified. This point may enable us to evaluate another clinical utility of furosemide compared with mannitol.

This study has several limitations. This is an open-label, single-institutional study. However, preplanned blood examination can provide some robustness regarding our primary endpoint. Second, considering patients' resources, we plan this study as a phase II. However, results obtained from 105 participants mean this will be the study with the most powerful evidence in this area. If the current study reaches its primary endpoint, it will suggest that furosemide may be non-inferior choice to mannitol.

Acknowledgements We deliver our thanks to Ms Keiko Fujimura and all the staffs for their valuable support in participant recruitment. Patient advisers and public were not involved in this study. We acknowledge English proofreading by Benjamin Phillis at the Clinical Study Support Center, Wakayama Medical University.

Contributors EM and HA designed the protocol and drafted the manuscript. TS performed the statistical analysis. KW and NY further aided in assessment and revision of the protocol and revised the manuscript. All authors read and approved the final version of the protocol.

Funding This trial was conducted with no external funding and was instead internally funded by the Department of Internal Medicine III, Wakayama Medical University.

Competing interests None declared.

Patient consent for publication Not required.

Ethics approval Institutional Review Board of Wakayama Medical University approved this study (approval number: 2258).

Provenance and peer review Not commissioned; externally peer reviewed.

Open access This is an open access article distributed in accordance with the Creative Commons Attribution Non Commercial (CC BY-NC 4.0) license, which permits others to distribute, remix, adapt, build upon this work non-commercially, and license their derivative works on different terms, provided the original work is properly cited, appropriate credit is given, any changes made indicated, and the use is non-commercial. See: http://creativecommons.org/licenses/by-nc/4.0/.

ORCID iD

Hiroaki Akamatsu http://orcid.org/0000-0001-5856-5512

\section{REFERENCES}

1 Sekine I, Kubota K, Tamura Y, et al. Innovator and generic cisplatin formulations: comparison of renal toxicity. Cancer Sci 2011;102:162-5.

2 Dobyan DC, Levi J, Jacobs C, et al. Mechanism of cis-platinum nephrotoxicity: II. morphologic observations. J Pharmacol Exp Ther 1980;213:551-6.

3 Cvitkovic E, Spaulding J, Bethune V, et al. Improvement of cisdichlorodiammineplatinum (NSC 119875): therapeutic index in an animal model. Cancer 1977;39:1357-61.

4 Pera MF, Zook BC, Harder HC. Effects of mannitol or furosemide diuresis on the nephrotoxicity and physiological disposition of cisdichlorodiammineplatinum-(II) in rats. Cancer Res 1979;39:1269-78.

5 Korogi Y, Yoshioka H, Kunimasa K, et al. Retrospective analysis of short-hydration cisplatin-based chemotherapy. Annals of The Japanese Respiratory Society 2013;2:730-6.

6 Ostrow S, Egorin MJ, Hahn D, et al. High-dose cisplatin therapy using mannitol versus furosemide diuresis: comparative pharmacokinetics and toxicity. Cancer Treat Rep 1981;65:73-8.

7 Santoso JT, Lucci JA, Coleman RL, et al. Saline, mannitol, and furosemide hydration in acute cisplatin nephrotoxicity: a randomized trial. Cancer Chemother Pharmacol 2003;52:13-18.

8 Horinouchi $\mathrm{H}$, Kubota $\mathrm{K}$, Itani $\mathrm{H}$, et al. Short hydration in chemotherapy containing cisplatin $(\geq 75 \mathrm{mg} / \mathrm{m} 2)$ for patients with lung cancer: a prospective study. Jpn J Clin Oncol 2013;43:1105-9.

9 Hotta K, Takigawa N, Hisamoto-Sato A, et al. Reappraisal of shortterm low-volume hydration in cisplatin-based chemotherapy: results of a prospective feasibility study in advanced lung cancer in the Okayama lung cancer Study Group trial 1002. Jpn J Clin Oncol 2013;43:1115-23. 